\title{
GROUPS WITH RESTRICTION ON THEIR INFINITE SUBNORMAL SUBGROUPS
}

\author{
by HERMANN HEINEKEN
}

(Received 26th August 1986)

Dedicated to B. Huppert on the occasion of his sixtieth birthday

\section{Introduction}

A group $G$ is called a $B_{n}$-group, if all its subnormal subgroups are of defect $n$. If all infinite subnormal subgroups of $G$ have defect $n$, we say that $G$ is an $I B_{n}$-group. DeGiovanni and Franciosi [2] have considered soluble $I B_{n}$ groups and characterized them.

The object of this note is the class of $I B_{n}$-groups in general, in particular in comparison to $B_{n}$-groups. We are able to show, that infinite $I B_{n}$-groups are extensions of an abelian group by a $B_{n}$-group (Proposition 1), where the structure of the abelian normal subgroup can be restricted (Proposition 2), furthermore infinite $I B_{n}$-groups are $B_{n+1}$-groups (Corollary 3 ).

Changing the notation we will call $B_{1}$-groups and $I B_{1}$-groups $T$-groups and $I T$ groups respectively. Gaschütz [3] considered finite $T$-groups while Robinson [5] was able to classify the soluble $T$-groups. De Giovanni and Franciosi [1] characterized soluble $I T$-groups. For IT-groups we have a result somewhat dual to Proposition 1: If $G$ is an infinite $I T$-group, it is an extension of a $T$-group by a residually finite $T$-group which in turn is metabelian or almost abelian of finite exponent (Proposition 3). Results for the soluble case are given separately. Finally, examples are given to show that some of the statements on IT-groups cannot be strengthened.

\section{Preparations}

We will make use of the following notation.

$$
X^{\tilde{J}}=\bigcap_{\substack{N \unlhd X \\|X: N| \text { finite }}} N
$$

$$
W(x)=\langle U| U \text { finite subnormal subgroup of } X\rangle
$$

If $T$ is a subnormal subgroup of $G$, we define the so-called canonical sequence

$$
T_{0}=G, T_{i+1}=T^{T_{i}} \quad \text { for all } i \geqq 0,
$$

moreover $T$ is of defect $n$ if and only if $T_{n}=T$. 
It is well known that $T_{k}$ is the intersection of all subnormal subgroups of defect $k$ in $G$ which contain $T$. So

$$
(A \cap B)_{k} \subseteq A_{k} \cap B_{k} \quad \text { for all } k .
$$

The following consequence will be needed.

$$
\text { If } x^{-1} A x=A \text { then } \quad x^{-1} A_{k} x=A_{k} \text { for all } k \geqq 0 \text {. }
$$

Looking for an abelian normal subgroup of restricted structure in $I B_{n}$-groups we require

\section{Lemma 1. Suppose}

(i) $G$ is an infinite $I B_{n}$-group

(ii) $A$ is a subnormal subgroup of $G$ which is not of defect $n$

(iii) $K \neq 1$ is a divisible abelian normal subgroup of $G$.

Then $K$ is a locally cyclic p-group for some prime $p$.

Proof. Hypotheses (i) and (ii) imply that $A$ is finite, on the other hand, (ii) and (iii) yield that $A K$ is an infinite subnormal subgroup of $G$. By (i),

$$
A_{n} \subseteq(A K)_{n}=A K
$$

Since $A$ is finite and subnormal in $A K$ and $K$ is divisible, we have in addition

$$
K \subseteq Z(A K)
$$

and, for every infinite subgroup $L$ of $K$,

$$
A_{n} \subseteq A L
$$

If $L$ is torsionfree, $A$ is the torsion subgroup of $A L$ and hence characteristic in $A L=(A L)_{n}$. This implies $A_{n}=A$, a contradiction. We have obtained

$$
K \text { is a torsion group. }
$$

Assume now that $K$ is not a $p$-group. Then there are subgroups $U \simeq C_{p^{\infty}}$ and $V \simeq C_{q^{\infty}}$ of $K$, where $p$ and $q$ are distinct primes.

Now

$$
A_{n} \subseteq A U \cap A V=A
$$

again a contradiction; this proves

$$
K \text { is a } p \text {-group for some prime } p \text {. }
$$


If $K$ is not locally cyclic, there is a divisible subgroup $Q$ of rank two in $K$. There are elements $b$ and $c$ in $Q$ such that

$$
A \cap Q=\langle b\rangle x\langle c\rangle,
$$

and we find subgroups $B$ and $C$ of $Q$ such that

$$
\begin{aligned}
& B C=Q \quad B \cap C=1 \\
& A \cap B=\langle b\rangle \text { and } A \cap C=\langle c\rangle \text {. }
\end{aligned}
$$

Now

$$
A_{n} \subseteq A B \cap A C=A,
$$

a final contradiction showing

$$
K \text { is locally cyclic. }
$$

Lemma 3 now follows from (2) and (3).

The next statement is probably well known, a proof is added for the convenience of the reader.

\section{Lemma 2. Suppose}

(i) $R$ is a finite subgroup of the subgroup $S$ of $G$.

(ii) $\mathscr{M}$ is a set of subgroups of $G$ such that

(a) $X \cap Y$ belongs to $\mathscr{M}$ if $X$ and $Y$ do,

(b) $S=R(S \cap X)$ for all $X$ in $\mathscr{M}$.

Then $S=R\left(S \cap \bigcap_{x \in \mathcal{M}} X\right)$.

Proof. The index $|S:(S \cap X)|=|R(S \cap X):(S \cap X)|=|R: R \cap X|$ is bounded by the order $|R|$ of $R$. So among the intersections $S \cap X$ with subgroups $X$ from $\mathscr{M}$ there is a minimal one, $S \cap Y$, say.

For all $X$ in $\mathscr{M}$ we have

$$
S \cap Y=S \cap(Y \cap X)=(S \cap Y) \cap X
$$

and

$$
\begin{gathered}
S \cap Y=(S \cap Y) \bigcap_{X \in \mathcal{M}} X=S \cap \bigcap_{X \in \mathcal{M}} X, \\
S=R(S \cap Y)=R\left(S \cap \bigcap_{X \in \mathcal{M}} X\right) .
\end{gathered}
$$




\section{Corollary 1. Suppose}

(i) $G$ is an infinite $I B_{n}$-group,

(ii) $A$ is a subnormal subgroup of $G$, not of defect $n$,

(iii) $L$ is an infinite subnormal subgroup of $G$ which is normalized by $A$.

Then $A_{n} \subseteq A L^{\mathfrak{s}}$.

Proof. From (i) and (ii) we deduce that $A$ is finite. The statement (+) implies that every $L_{i}$ is normalized by $A$, so $A L$ is an infinite subnormal subgroup of $G$ and $A_{n} \subseteq A L$.

Every normal subgroup $K$ of finite index in $L$ contains an $A$-invariant normal subgroup $K^{*}$ of $L$ which is still of finite index in $L$. We conclude $A_{n} \subseteq A K^{*}$ and $A_{n}=A\left(A_{n} \cap K^{*}\right)=A\left(A_{n} \cap K\right)$.

We apply Lemma 2 for $R=A$ and $S=A_{n}$, while $\mathscr{M}$ is the set of all normal subgroups of finite index in $L$; now Corollary 1 follows.

\section{The abelian normal subgroup}

In a first step, we find an abelian normal subgroup of our $I B_{n}$-group $G$ with a $B_{n}$ group as quotient group. Later on refinements lead to the abelian normal subgroup of restricted structure.

Proposition 1. If $G$ is an infinite $I B_{n}$-group and $A$ is a subnormal subgroup of $G$, then $A Z(W(G))$ is subnormal of defect $n$.

Proof. $A Z(W(G))$ is subnormal of the same defect as $A$ since $Z(W(G))$ is a characteristic subgroup of $G$. Therefore the statement of the Proposition is nontrivial for finite subnormal subgroups only. Denote by $V$ any finite subnormal subgroup of $G$. By Wielandt [6; statement (10), p. 218] the subgroup $R=\langle A, V\rangle$ is also finite and subnormal in $G$. Considering the canonical sequence for $R$, we see that there is an index $i$ such that $R_{i}$ is infinite while $R_{i+1}$ is finite. Since $R_{i+1}$ is normal in $R_{i}$, we conclude that $C\left(R_{i+1}\right) \cap R_{i}$ is an infinite subnormal subgroup and

$$
A_{n} \subseteq A\left(C\left(R_{i+1}\right) \cap R_{i}\right)
$$

Thus

$$
A_{n}=A\left(A_{n} \cap C\left(R_{i+1}\right)\right)=\left(A_{n} \cap C(R)\right)=A\left(A_{n} \cap C(V)\right) .
$$

If $U$ and $V$ are two finite subnormal subgroups of $G$, the subgroup $\langle U, V\rangle$ is also finite and subnormal and

$$
C(U) \cap C(V)=C(\langle U, V\rangle)
$$

We apply Lemma 2 to $A, A_{n}$, and the set $\mathscr{M}$ of all centralizers of finite subnormal 
subgroups and find

$$
A_{n}=A\left(A_{n} \cap C(W(G))=A\left(A_{n} \cap Z(W(G))\right)\right.
$$

since $A_{n} \subseteq W(G)$.

Now Proposition 1 follows.

Corollary 2. If $G$ is an infinite $I B_{n}$-group and not a $B_{n}$-group, then $Z(W(G))$ is finite or the direct product of a finite group with a group of Prüfer type.

Proof. Consider the set $S$ of all elements of square-free order in $Z(W(G))$. This is a normal subgroup of $G$, not of defect $n$. Then, by Corollary 1 ,

$$
A_{n} \subseteq A S^{\mathscr{J}}=A,
$$

a contradiction. We have

(1) The socle $S$ of $Z(W(G))$ is finite.

Applying Lemma 1 to the divisible part of $Z(W(G))$ yields

(2) the divisible part of $Z(W(G))$ is trivial or a locally cyclic $p$-group.

Now the Corollary follows.

Corollary 3. If $G$ is an infinite $I B_{n}$-group and $A$ is a subnormal subgroup of $G$, then

(i) $A^{\prime}$ is subnormal of defect $n$,

(ii) $A$ is of defect $n+1$,

(iii) $A$ is of finite index in $A_{n}$.

Proof. All three statements are nontrivial only for those subnormal subgroups $A$ which are not of defect $n$.

For this case we deduce from Proposition 1

$$
A_{n}=A\left(A_{n} \cap Z(W(G))\right)=A Z\left(A_{n}\right)
$$

Now $A$ is normal in $A_{n}$ and $A_{n}^{\prime}=\left(A Z\left(A_{n}\right)\right)^{\prime}=A^{\prime}$.

This shows (i) and (ii).

By (ii) we know that $A_{n}$ is generated by some conjugates of $A$ which are all normal in $A_{n}$. We deduce that the exponents of $A_{n} /\left(A_{n}\right)^{\prime}$ and of $A / A^{\prime}$ are the same and the exponent of $A_{n}$ is finite.

Now $Z\left(A_{n}\right)^{g}=1$, and we deduce from Corollary 1 that $Z\left(A_{n}\right)$ cannot be infinite. Now (iii) follows easily.

Corollary 3(ii) generalizes results of De Giovanni and Franciosi [2; Corollary]. We can now do our final reduction. 
Proposition 2. If $G$ is an infinite $I B_{n}$-group, there exists an abelian normal subgroup $\dddot{K}$ of $G$ such that

(i) $G / K$ is a $B_{n}$-group,

(ii) $K$ is finite or a group of Prüfer type.

Proof. We have to choose $K \neq 1$ only if $G$ is not a $B_{n}$-group. In this case, by Proposition 1, we know that $A Z(W(G))$ is subnormal of defect $n$ for all subnormal subgroups $A$ of $G$. If $Z(W(G))$ is finite, we use $K=Z(W(G))$ for Proposition 2.

If $Z(W(G))$ is infinite, we use Corollary 1 and find that $A\left(Z(W(G))^{\mathfrak{f}}\right.$ is subnormal of defect $n$ for all subnormal subgroups $A$ of $G$. We take $K=(Z(W(G)))^{\text {s }}$ since this is a group of Prüfer type by Corollary 2.

Proposition 2 should be compared with results of De Giovanni and Franciosi [1, Theorem 1.11 and 2, Theorems A and B]: In the soluble case $K$ can be chosen of Prüfer type.

\section{Normal $T$-subgroups of $I T$-groups}

Before we looked for a comparatively small normal subgroup $A$ of the $I B_{n}$-group $G$ such that $G / A$ is a $B_{n}$-group. In this section we want to construct another normal subgroup $M$ which is itself a $T$-group and whose quotient group $G / M$ is restricted. The form of restriction depends on the abelian normal subgroup mentioned in Proposition 2.

Proposition 3. Assume that $G$ is an infinite IT-group and $A$ is an abelian normal subgroup of $G$ such that $G / A$ is a $T$-group. Then:

(a) There is a normal $T$-subgroup $M$ of $G$ such that $G / M$ is a $T$-group and residually finite.

(b) If $A$ is finite of exponent $r, G / M$ is an extension of an abelian group of exponent $r$ by a finite group,

(c) If $A$ is cyclic or locally cyclic, $G / M$ is metabelian.

Proof. If $S$ is any subnormal subgroup of $G$, it is either normal or contained in a finite normal subgroup of $G$. So in any case $N_{G}(S)$ is of finite index in $G$. We deduce that

$$
M=\bigcap_{S \rightarrow \triangle G} N_{G}(S)
$$

is a normal subgroup with quotient group $G / M$ residually finite. $M$ is a $T$-group by construction. Since

$$
Z(W(G)) \subseteq N_{G}(S) \quad \text { for all } S \triangleleft \triangleleft G,
$$

we have $Z(W(G)) \subseteq M$, and $G / M$ is a $T$-group by Proposition 1 . So we have shown (a). 
For statements (b) and (c) we have to consider the quotient group

$$
G / \bigcap_{g \in G} g^{-1} N_{G}(S) g
$$

for some non-normal subnormal subgroup $S$ of $G$.

Since $S$ is subnormal and $G / A$ is a $T$-group, $A S$ is a normal subgroup of $G$ and $A S=A S^{G}$. We deduce from the modular law $S^{G}=S K$, where $K=A \cap S^{G}$. Assume $r=\exp K$. We have seen earlier that $\left(S^{G}\right)^{\prime}=S^{\prime}$, using this we obtain

$$
\left(S^{G}\right)^{\prime}\left(S^{G}\right)^{r}=\left(S^{G}\right)^{\prime} S^{r} K^{r}=S^{\prime} S^{r}
$$

and $S^{\prime} S^{r}$ is a normal subgroup of $G$.

Let

$$
D_{S}=C(A) \cap\left\{x \mid[x, y] \in S^{\prime} S^{r} \text { for all } y \in S^{G}\right\}
$$

and

$$
E_{S}=C(A) \cap\left\{x \mid[x, y] \in S^{\prime} S^{r} K \text { for all } y \in S^{G}=S K\right\} .
$$

The elements of $C(A)$ induce in $S K / S^{\prime} S^{r} K \simeq S A / S^{\prime} S^{r} A$ only power automorphisms since $G / A$ is a $T$-group.

We deduce

$$
C(A) / E_{S} \text { is abelian and isomorphic to a subgroup of } \operatorname{Aut}\left(C_{r}\right) .
$$

On the other hand, the elements of $E_{S}$ stabilize the chain

$$
S K \supseteq S^{\prime} S^{r} K \supseteq S^{\prime} S^{r}
$$

and

$$
E_{S} / D_{S} \text { is abelian of exponent } r \text {. }
$$

Now if $A$ is finite, $C(A)$ is of finite index in $G$, and we have

If $A$ is finite of exponent $r, G / D_{S}$ is an extension of an abelian group of exponent $r$ by a finite group,

If, on the other hand, $A$ is cyclic or locally, cyclic, $G / C(A)$ is abelian, and

$$
\text { If } A \text { is cyclic or locally cyclic, } G / D_{S} \text { is soluble. }
$$

In both cases (a) and (b) we have $C(A) \supseteq Z(F C(G))$ and therefore

$$
D_{s} \supseteq Z(F C(G)),
$$


so

$$
D=\bigcap_{S \subseteq G} D_{S}
$$

is a normal subgroup with $T$-quotient group $G / D$. From (3a) and (3b) we obtain

If $A$ is finite of exponent $r, G / D$ is an extension of an abelian group of exponent $r$ by a finite group

and

If $A$ is cyclic or locally cyclic, $G / D$ is metabelian.

Now $D_{S} \supseteq \bigcap_{g \in G} g^{-1} N(S) g$, and so $D \subseteq M$. This shows (a) and (b).

\section{Soluble $I T$-groups}

Closer knowledge of soluble IT-groups allows the strenthening of the results for. this special case.

Proposition 4. If $G$ is an infinite soluble IT-group, then $G$ possesses a normal $T$ subgroup of finite index.

Proof. If $G$ is periodic, $G$ is abelian by finite (see De Giovanni and Franciosi [1, Theorem 1.10]), so the statement is true.

If $G$ is nonperiodic, $W(G)$ is an extension of a Prüfer group $A \simeq C_{p_{\infty}}$ by a finite abelian group (see [1, Theorem 1.11]).

All non-normal subnormal subgroups of $G$ are subgroups of $W(G)$, and finite subnormal subgroups $S$ of order prime to $p$ are normal since they are characteristic subgroups of the normal subgroup $A S$.

The maximal $p$-subgroup of $W(G)$ is then generated by $A$ and some finite $p$-group $L$ of exponent, say, $p^{k}$. If $A^{+}$is the subgroup of order $p^{k}$ of $A, A^{+} L$ is a characteristic subgroup of $A L$ and hence normal in $G$, and $C\left(A^{+} L\right)$ is a normal subgroup of finite index in $G$. In order to show that $C\left(A^{+} L\right)$ is a $T$-group it suffices to show that subnormal subgroups $K$ of $G$ which are contained in $A L$ are normal in $C\left(A^{+} L\right)$. Now $K^{p^{k}}$ is contained in $(A L)^{p^{k}}=A$ and is normal in $G$.

Choose $B$ in $A$ such that $B^{p^{k}}=K^{p^{k}}$. Then $K \subseteq B L$ and $\left[B L, C\left(A^{+} L\right)\right] \subseteq B^{p^{k}} \leqq K^{p^{k}}$. This shows that $K$ is normalized by $C\left(A^{+} L\right)$ and $C\left(A^{+} L\right)$ is a $T$-group.

Proposition 5. If $G$ is an infinite soluble IT-group, then $G$ possesses a finite normal subgroup $U$ such that $G / U$ is a $T$-group.

Proof. It follows from [1, Theorem 1.10 and 1.11] that $W(G)$ is the extension of a Prüfer group $A$ by finite group. Since $A \subseteq Z(W(G))$, and $A$ is a subgroup of finite index, by a well-known theorem of Schur we have that $(W(G))^{\prime}$ is finite, and there is a finite characteristic subgroup $R$ of $W(G)$ such that $W(G) / R$ is a Prüfer subgroup. If $X / R$ is a 
non-normal subnormal subgroup of $G / R$, then $X$ is also non-normal and subnormal in $G$ and contained in $W(G)$. Now $X / R$ is a subgroup of $W(G) / R$ which is a normal subgroup possessing characteristic subgroups only. So $G / R$ is a $T$-group.

\section{Examples}

Our first example is an IT-group which is a $W$-group and no quotient group modulo $a$ finite normal subgroup is a $T$-group; also no normal subgroup of finite index is a $T$-group.

Example 1. Let $p$ be a prime. For every natural number $i$ we choose a pair $X_{i}, Y_{i}$ of groups such that

$$
\begin{gathered}
X_{i}^{\prime} \supset Z\left(X_{i}\right), Y_{i}^{\prime} \supset Z\left(Y_{i}\right), \\
X_{i}^{\prime} / Z\left(X_{i}\right) \text { and } Y_{i}^{\prime} / Z\left(Y_{i}\right) \text { are nonabelian simple, } \\
X_{i} / X_{i}^{\prime} \simeq Y_{i} / Y_{i}^{\prime} \simeq C_{p^{i+1}}, Z\left(X_{i}\right) \simeq Z\left(Y_{i}\right) \simeq C_{p^{i}}
\end{gathered}
$$

(If $q^{t}-1$ is divisible by $p^{i+1}$, a quotient group of a normal subgroup of $G L\left(p^{i+1}, p^{t}\right.$ ) will do for $X_{i}$ and $Y_{i}$ ).

Let

$$
X_{i}=\left\langle X_{i}^{\prime}, x_{i}\right\rangle, \quad Y_{i}=\left\langle Y_{i}^{\prime}, y_{i}\right\rangle, \quad Z\left(X_{i}\right)=\left\langle u_{i}\right\rangle, \quad Z\left(Y_{i}\right)=\left\langle v_{i}\right\rangle,
$$

and consider

$$
W_{i}=\left\langle s_{i}, t_{i} \mid s_{i}^{p^{i+1}}=t_{i}^{p^{i+1}}=\left[\left[s_{i}, t_{i}\right], t_{i}\right]=\left[\left[s_{i}, t_{i}\right] s_{i}\right]=1\right\rangle .
$$

We have $Z\left(W_{i}\right)=\left\langle\left[s_{i}, t_{i}\right]\right\rangle \simeq C_{p^{i+1}}$. We form the direct product $X_{i} \times Y_{i} \times W_{i}$ and choose a quotient group of some subgroup, namely

$$
D_{i}=\left\langle X_{i}^{\prime}, Y_{i}^{\prime}, x_{i} s_{i}, y_{i} t_{i}\right\rangle\left\langle\left\langle\mu_{i} v_{i}, u_{i}\left[s_{i}, t_{i}\right]^{p}\right\rangle .\right.
$$

Again $D_{i}$ has a cyclic centre of order $p^{i+1}$, it is generated by the image $d_{i}$ of $\left[s_{i}, t_{i}\right]$.

The IT-group we want to construct is

$$
G=\left(D_{1} \times D_{2} \times \cdots\right) /\left\langle d_{1}^{-1} d_{2}^{p}, \ldots, d_{i}^{-1} d_{i+1}^{p}, \ldots\right\rangle
$$

$G / Z(G)$ is a $T$-group and $Z(G)$ is a Prüfer group. If $L$ is a finite normal subgroup of $G$, there is an integer $m$ such that $D_{m}$ is not mapped onto a $T$-group under the canonical mapping of $G$ onto $G / L$. A corresponding statement holds for normal subgroups of finite index. The commutator subgroup $G^{\prime}$ is a $T$-group, for instance. (The groups $D_{i}$ are counterexamples to Satz 5 of [3]. Its incorrectness was noticed earlier by G. Stroth, see U. Kassens [4, p. 3 and Beispiel 4.19, p. 54].) 
The following is an example of an IT-group which is a $F C$-group such that the quotient group modulo $a$ finite normal subgroup is a $T$-group.

Example 2. The central product of a finite extra-special 2-group with infinitely many groups isomorphic to $S L(2,5)$ is an $I T$-group. Modulo its centre of order 2 it is a $T$-group. Its maximal perfect subgroup is a normal $T$-subgroup of finite index.

Our last example shows that the finite abelian normal subgroup $B$ in Proposition 2 can be of any form, also the quotient group $G / M$ mentioned in Proposition 3 can be any finite $T$-group.

Example 3. Let $H$ be a finite $T$-group and a subgroup of the Galois group of some normal extension $F$ of the field $Q$ of rational numbers. We denote by $L$ the group of all invertible linear mappings of an $F$-vector space of countably infinite dimension onto itself, and by $L^{*}$ the set of those mappings fixing a subspace of finite codimension elementwise.

Then $L^{+}=L^{\prime} L^{*} / L^{*}$ is simple modulo its centre, and $Z\left(L^{*}\right)$ is isomorphic to the multiplicative group of $F$.

Once a basis of the vector space is fixed, there is a canonical extension $K$ of $L^{+}$by $H$, using the automorphism description of every element in $H$. We consider $H$ and $L^{+}$as subgroups of $K$, and obtain

$$
F C(K)=Z\left(L^{+}\right)
$$

If $T \triangleleft K$ is of finite index in $F C(K)$, the quotient group $K / T$ is an $I T$-group, further $L^{+} / T$ is a normal $T$-subgroup of $K / T$ such that

$$
(K / T)\left(L^{+} / T\right) \simeq K / L^{+}=H .
$$

On the other hand, $K / T$ possesses a finite abelian normal subgroup $W(K) / T$ such that

$$
(K / T) /(W(K) / T) \simeq K / W(K)
$$

is a $T$-group.

Close analysis of the construction method will show that even the way $H$ operates on $W(K) / T$ can be prescribed, once the field $F$ possesses a correspondingly big Galois group.

\section{REFERENCES}

1. F. De Glovanni and S. Franciosi, Groups in which every infinite subnormal subgroup is normal, J. Algebra 96 (1985), 566-580.

2. F. De Glovanni and S. Franciosi, Groups with infinite subnormal subgroups of bounded defect, Boll. Un. Mat. Ital. D(6) 4 (1985), 49-56.

3. W. GaschUtz, Gruppen, in denen das Normalteilersein transitiv ist, J. Reine. Angew. Math. 198 (1957), 87-92. 
4. U. KASSENS, Die Wielandtlänge endlicher Gruppen (Hausarbeit zur Lehramtsprüfung, F.U. Berlin, 1980).

5. D. J. S. Rosinson, Groups in which normality is a transitive relation, Proc. Cambridge Philos. Soc. 60, (1964), 21-38.

6. H. WIELANDT, Eine Verallgemeinerung der invarianten Untergruppen, Math. Z. 45 (1939), 209-244.

Mathematisches INSTITUT

UNIVERSITÄT WURZBURG

Federal Republic of Germany 